IGCS20_1233

\section{SEARCH FOR PREDICTIVE BIOMARKERS OF SENSITIVITYI RESISTANCE AGAINST CISPLATIN IN HIGH-GRADE SEROUS OVARIAN CANCER}

${ }^{1} \mathrm{~A}$ Cortez ${ }^{*},{ }^{2} \mathrm{~A}$ Glodek, ${ }^{1} \mathrm{~A}$ Krzywon, ${ }^{2} \mathrm{~J}$ Polanska, ${ }^{1} \mathrm{KM}$ Lisowska. ${ }^{1}$ Maria Sklodowska-Curie National Research Institute Of Oncology Gliwice Branch, Poland; ${ }^{2}$ Silesian University of Technology, Poland

10.1136/ijgc-2020-IGCS.196

Our aim was to search for potential predictive biomarker(s) of cisplatin resistance in high-grade serous ovarian cancer (HGSOC). We have re-analyzed the data published by Koti et al. ${ }^{1}$

Data encompassed 28 HGSOC gene expression profiles obtained using Affymetrix U133-Plus-2.0-GeneChips. Based on progression free survival (PFS) after first-line chemotherapy, 16 samples were classified as platinum-sensitive (PFS $>18$ months), 12 as platinum-resistant $(\mathrm{PFS}<6)$. Data was filtered using Gaussian Mixture Modeling decomposition, ${ }^{2}$ in order to select transcripts that were characterized by large value of variance. Normality of data distribution was checked with Shapiro-Wilk test. Because of non-normal distribution U MannWhitney Test was performed and U Mann Whitney effect size was calculated for up- and down-regulated transcripts. Transcripts with $\mathrm{p}$-values $<0,001$ and large or very large effect size were considered significant.

Our approach resulted in 356 probe-sets. Based on literature review we selected 40 potential candidate biomarkers for further validation. Selected probe-sets correspond to proteins related with mitochondrial function, nuclear- and cellularmembrane transport, that are processes related with drug resistance.

Our computational approach resulted in a larger list of probe-sets than that of Koti et al. ${ }^{1}$ In our opinion, their analysis suffered from the combination of parametric and nonparametric tests, inappropriately used for the data with nonnormal distribution. Using non-parametric test suited for nonnormal distributed dataset and analysis of effect size allowed to receive more reliable results, although selected candidate biomarkers must be further validated.

A.J.Cortez and A.Glodek were co-financed by the EU through the European Social Fund (grant-POWR.03.02.00-00I029).

\section{REFERENCES}

1. Koti M, et al. 2013,DOl:10.1186/1471-2407-13-549

2. Polanski A, et al. 2015,DOl:10.1371/journal.pone.0134256

\section{IGCS20_1234}

\section{UTILIZATION OF IMMUNOTHERAPY AMONG ELIGIBLE PATIENTS WITH CERVICAL CANCER}

${ }^{1} \mathrm{M}$ Smith*, ${ }^{2} \mathrm{~B}$ Pothuri. ${ }^{1} \mathrm{NYU}$ Grossman School of Medicine, USA; ${ }^{2}$ Department of Obstetrics and Gynecology, NYU Langone Health, USA

\subsection{6/ijgc-2020-IGCS.197}

Introduction Pembrolizumab, a checkpoint inhibitor with a favorable risk/benefit profile, is FDA approved for recurrent or progressive PD-L1 positive cervical cancer treated with prior chemotherapy. The use of pembrolizumab in clinical practice after approval has not been extensively studied. The purpose of this study is to determine frequency of pembrolizumab use among eligible patients with cervical cancer.

Methods This retrospective study includes all patients who underwent surgical procedure or radiation therapy for cervical cancer at an urban private and public hospital system between $5 / 1 / 2012$ and 1/31/2019 with recurrent or progressive disease after chemotherapy diagnosed after $7 / 12 / 18$ (date of FDA approval) and PD-L1 positive tumor. Eligible patients were recorded as having received or not received pembrolizumab.

Results Of the 211 cervical cancer patients screened, 21 presented after the date of FDA approval with recurrent or progressive disease and received prior chemotherapy. Twelve (57.1\%) patients received PD-L1 testing and 9 (42.9\%) potentially eligible patients did not. Eleven patients had PD-L1 positive tumors and were eligible for pembrolizumab. Of eligible patients, 7 (63.6\%) received pembrolizumab and 4 (36.4\%) did not; 1 was enrolled in a clinical trial and 3 received chemotherapy.

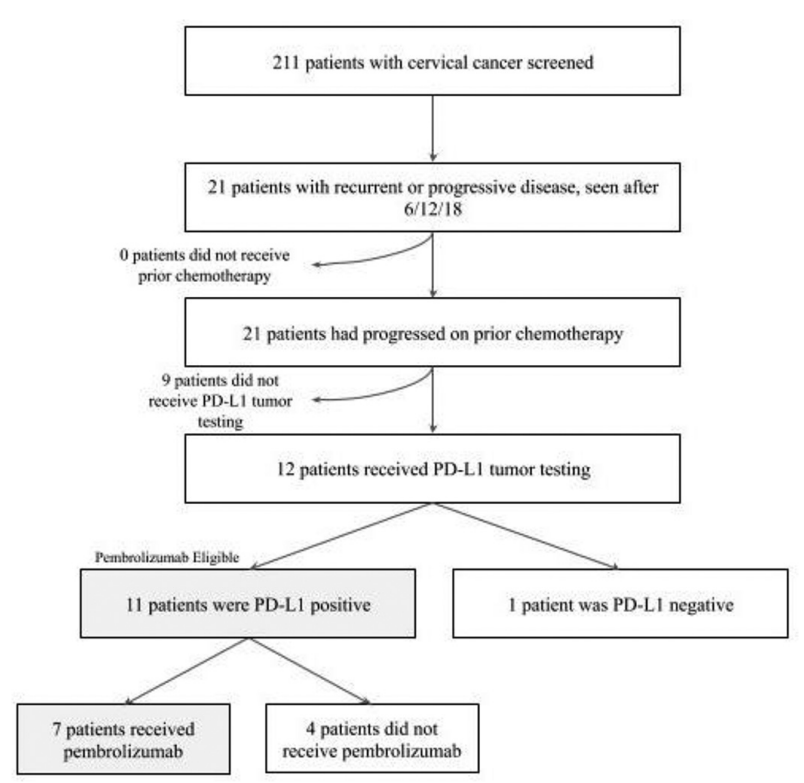

Abstract 231 Figure 1

Conclusion Immunotherapy is underused in patients with advanced cervical cancer. Over $40 \%$ of patients with progressive/recurrent cervical cancer don't even undergo testing for PD-L1 expression; in those with positive PD-L1 expression only two thirds of patients received pembrolizumab, an FDA approved therapy. There is an opportunity to improve the identification of eligible patients and increase the use of checkpoint inhibitors in cervical cancer.

\section{IGCS20_1235}

\section{UNRAVELLING CERVICAL ADENOSARCOMA}

R Rivera*, J Bagadiong. JRRMMC, Philippines

10.1136/ijgc-2020-IGCS. 198 